\title{
Análisis del control de emisiones atmosféricas vehiculares en Lima Metropolitana
}

Analysis of the control of vehicular atmospheric emissions in Metropolitan Lima

\author{
Christel Pinedo-Jáuregui ${ }^{1,2}{ }^{\oplus}$, José Verano-Cachay ${ }^{1,2 *}{ }^{\bullet}$ y \\ Víctor Barrantes-Santos ${ }^{1 \oplus}$
}

1 Facultad de Ciencias Ambientales, Universidad Científica del Sur. Lima, Perú.

\section{SOUTH} SUSTAINABILITY

Citar como: Pinedo-Jáuregui, C., Verano-Cachay, J., Barrantes-Santos, V. (2020). «Análisis del control de emisiones atmosféricas vehiculares en Lima Metropolitana». South Sustainability, 1(1), e005 DOI: 10.21142/SS-0101-2020-005

Artículo recibido: 15/12/2019 Arbitrado por pares Artículo aceptado: 25/3/2020

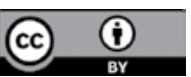

(C)Los autores 2020. Publicado por la Universidad Científica del Sur (Lima, Perú)

${ }^{*} E$-mail de correspondencia: jveranocachay@gmail.com

2 Ambos autores contribuyeron por igual al presente trabajo.

\section{RESUMEN}

La calidad del aire en Lima es un tema muy discutido, sobre todo por su estrecha relación con las emisiones vehiculares. Por ello, se han implementado diversas medidas de control y prevención para los automóviles, como el Sistema Nacional de Inspecciones Técnicas Vehiculares (ITV), que determina los automotores aptos para circular de manera segura por cumplir con las condiciones especificadas en la normativa vigente, $y$, además, comprueba que el nivel de emisiones no supere los límites máximos permisibles. Por eso es obligatorio el certificado de inspección vehicular (CITV), que autoriza la circulación del vehículo. En el marco de las nuevas políticas de mejora de la calidad de aire, la Municipalidad Metropolitana de Lima (MML) ejecutó una campaña de sensibilización en 10 puntos de la capital, con el objetivo de concientizar a los conductores de vehículos livianos sobre la importancia del buen mantenimiento del motor para reducir las emisiones vehiculares y medir sus emisiones vehiculares. Esta investigación analiza los datos recogidos en esa campaña. En el diagnóstico se resalta que el $79 \%$ de los vehículos evaluados incumplieron la normativa, a pesar de que la mayoría contaban con el ITV vigente, lo que muestra la falta de veracidad en los datos. El parámetro con más incumplimiento en esta investigación fue el del porcentaje de monóxido de carbono y dióxido de carbono, seguido del parámetro de hidrocarburos. Esta situación expone la combustión incompleta de los motores vehiculares, que da a lugar a las emisiones de monóxido de carbono e hidrocarburos.

Palabras clave: Control, emisiones vehiculares, límites máximos permisibles, inspección técnica, Lima

\section{ABSTRACT}

Air quality in Lima is a widely discussed topic, particularly with regard to its close association with vehicle emissions. Consequently, a number of automobile control and prevention measures have been implemented, such as the national Vehicle Technical Inspection (VTI) system. The national VTI system determines which cars are suitable for safe driving, in compliance with certain conditions specified by current regulations, while verifying that emissions levels do not exceed the maximum permissible limits. Thus, obtaining a vehicle inspection certificate (VTIC), authorizing the circulation of a vehicle, is now mandatory. Within the framework of new policies to improve air quality, the Metropolitan Municipality of Lima (MML) implemented an awareness campaign at ten locations throughout the city, with the aim of raising awareness among drivers of light vehicles regarding the importance of proper engine maintenance, in order to reduce vehicle emissions, as well as to measure those emissions. This research analyzes the data collected during that campaign, concluding that $79 \%$ of the vehicles evaluated as part of the campaign were found to violate regulations, despite the fact that most of the vehicles had a valid VTI, thereby exposing the lack of veracity in the data gathered. Our research found that the parameter most frequently violated was the percentage of carbon monoxide and carbon dioxide, followed by the hydrocarbon parameter, a situation exposing the incomplete combustion of vehicle engines, giving rise to emissions of carbon monoxide and hydrocarbons.

Keywords: Control, vehicle emissions, maximum permissible limits, technical inspection, Lima 


\section{Introducción}

A nivel global, el 54 \% de la población vive en ciudades. En el caso del Perú, esta cifra crece al 78,2 \% (Zucchetti y Freundt, 2018). La cantidad creciente de población en las zonas urbanas agregó al debate sobre la sostenibilidad la necesidad encarecida de pensar en políticas para el desarrollo de ciudades inteligentes y sostenibles. Ciudad sostenible es un concepto que se enfoca en los resultados técnicos hacia una transformación urbana más efectiva, basada en los impactos de sostenibilidad (Macke, Rubim Sarate y De Atayde Moschen, 2019). El Perú tiene como objetivo el cumplimiento de los compromisos de desarrollo sostenible suscritos en la Convención Marco de las Naciones Unidas sobre Cambio Climático (CMNUCC). Uno de estos compromisos es el Objetivo 11, Ciudades y Comunidades Sostenibles, que tiene el propósito de lograr que las ciudades y los asentamientos humanos sean seguros, resilientes y sostenibles. Este objetivo se divide en objetivos específicos, entre los que resalta el 11.2, dirigido a proporcionar sistemas de transporte seguros, económicos y sustentables para todos los ciudadanos, y así aumentar la seguridad vial, a fin de lograr una ampliación del servicio de transporte público con especial atención a las necesidades de poblaciones vulnerables (ONU, 2018).

En las grandes urbes del mundo, una de las principales fuentes de contaminación atmosférica es el parque automotor (Caminos et al., s. f.). En las últimas décadas se demostró la relación directa entre la flota vehicular y la contaminación atmosférica, debido sobre todo a la generación de gases emitidos por una combustión incompleta en el motor (Vintimilla, 2015). Por ese motivo, se crean instrumentos de gestión y control de emisiones. En el Perú se tomó conciencia de lo urgente que es aplicar políticas para mejorar la calidad del aire $y$, de este modo, controlar las principales fuentes como el parque automotor. Desde 2012, el parque automotor en el Perú ha crecido $7 \%$ en promedio. En 2016 cerca del $46 \%$ del parque automotor era representado por automóviles livianos (Posada, 2018). Por eso es obligatorio que los vehículos en circulación cuenten con un CITV, que valide el buen estado del motor y que cumpla con la normativa vigente en relación con los límites máximos permisibles para emisiones de gases vehiculares.

Esta investigación analiza los datos recogidos por la campaña «Respira Limpio» ejecutada por la MML del 8 de agosto al 17 de setiembre de 2019 en 10 puntos de la capital. El análisis abarca la comparación de los valores de emisiones de gases vehiculares registrados durante la campaña y los valores correspondientes a su CITV. La campaña tuvo como objetivo concientizar a los conductores de vehículos livianos sobre la importancia del buen mantenimiento del motor para reducir las emisiones vehiculares y, al mismo tiempo, cuidar el vehículo.

En el Perú se tomó conciencia de que una de las principales fuentes contaminantes de la calidad del
Tabla 1. Límites máximos permisibles $M$ y N para vehículos en circulación

\begin{tabular}{|c|c|c|c|}
\hline \multirow{2}{*}{\multicolumn{2}{|c|}{$\begin{array}{l}\text { Parámetros categoría M y N } \\
\text { (0 a } 1800 \mathrm{msnm})\end{array}$}} & \multicolumn{2}{|c|}{$\begin{array}{l}\text { Decreto Supremo } \\
\text { 010-2017-MINAM }\end{array}$} \\
\hline & & Gasolina & GNV/ GLP \\
\hline \multirow{3}{*}{ Hasta 1995} & $\mathrm{CO}(\%)$ & 3 & 3 \\
\hline & $\mathrm{HC}(\mathrm{ppm})$ & 400 & 400 \\
\hline & $\mathrm{CO}+\mathrm{CO}_{2}(\%)$ mínimo & 10 & 8 \\
\hline \multirow{3}{*}{1996 a 2002} & $\mathrm{CO}(\%)$ & 2,5 & 2,5 \\
\hline & $\mathrm{HC}(\mathrm{ppm})$ & 300 & 300 \\
\hline & $\mathrm{CO}+\mathrm{CO}_{2}(\%)$ mínimo & 10 & 8 \\
\hline \multirow{3}{*}{$\begin{array}{l}2003 \text { en } \\
\text { adelante }\end{array}$} & $\mathrm{CO}(\%)$ & 0,5 & 0,5 \\
\hline & $\mathrm{HC}(\mathrm{ppm})$ & 100 & 100 \\
\hline & $\mathrm{CO}+\mathrm{CO}_{2}(\%)$ mínimo & 12 & 8 \\
\hline
\end{tabular}

Fuente: Recuperado del Decreto Supremo 010-2017-MINAM para vehículos de categorías $M$ y $\mathrm{N}$ con motor de encendido por chispa a gasolina, GLP o GNV como combustible u otros combustibles alternos.

aire son las emisiones vehiculares y, por ello, se crearon instrumentos normativos que permitieran su control. El Decreto Supremo 047-2001-MTC estableció a nivel nacional los límites máximos permisibles para emisiones de vehículos automotores en circulación, nuevos y usados importados, y los que serán ensamblados en el país. Posteriormente se realizaron varias modificatorias, de las que resultó el vigente Decreto Supremo 010-2017-MINAM, que establece límites máximos permisibles de emisiones atmosféricas para vehículos automotores (ver tabla 1). Este decreto fue previamente presentado a la Comisión Multisectorial para la Gestión de la Iniciativa del Aire Limpio para Lima y Callao, a fin de recibir comentarios y aportes. Paralelamente se creó el Grupo de Trabajo Multisectorial, encargado de proponer medidas para mejorar la calidad del aire a nivel nacional vinculadas a las emisiones vehiculares. Entre las propuestas está lo establecido en el Decreto Supremo 025-2017-EM del Ministerio de Energía y Minas, que regula la reducción del contenido de azufre por debajo de los 50 ppm en la gasolina y el gasohol (Decreto Supremo 025, 2017, artículo 2).

\section{Materiales y métodos}

\section{Población y muestra}

La MML ejecutó la campaña de sensibilización «Respira Limpio» en coordinación con la empresa Touring y Automóvil Club del Perú en 10 puntos de la capital (ver tabla 2). El objetivo fue medir las emisiones atmosféricas del tubo de escape en vehículos livianos (categoría M) y concientizar a los conductores sobre las emisiones atmosféricas de su vehículo y el mantenimiento del motor. La campaña contó con la participación de 327 conductores, quienes voluntariamente sometieron su vehículo a la evaluación. 
Tabla 2. Puntos de ejecución de la campaña «Respira Limpio»

\begin{tabular}{|c|c|c|c|c|}
\hline Nro. & Distrito & Ubicación & Fecha & Horario \\
\hline 1 & Villa María del Triunfo & $\begin{array}{c}\text { Av. Salvador Allende con av. } 26 \text { de Noviembre } \\
\text { (frente al Hospital Kaelin) en Villa María del Triunfo }\end{array}$ & 8 de agosto de 2019 & 11:00 p. m.-1:00 p. m. \\
\hline 2 & Jesús María & Av. Salaverry, cdra. 1 & 23 de agosto de 2019 & 9:00 a. m.-12:00 m. \\
\hline 3 & San Isidro & Av. Petit Thouars, cruce con av. Javier Prado & 26 de agosto de 2019 & 9:00 a. m.-12:00 m. \\
\hline 4 & Santa Anita & Av. Ruiseñores, cruce con av. Huancaray & 27 de agosto de 2019 & 9:00 a. m.-12:00 m. \\
\hline 5 & La Molina & Av. La Fontana, cdra. 10 & 28 de agosto de 2019 & 9:30 a. m.-12:30 p. m. \\
\hline 6 & Villa El Salvador & Pastor Sevilla, cruce con av. El Sol & 2 de setiembre de 2019 & 9:00 a. m.-12:00 p. m. \\
\hline 7 & Surquillo & Av. Angamos Este, cruce con av. Tomás Marsano & 3 de setiembre de 2019 & 9:00 a. m.-12:00 m. \\
\hline 8 & Miraflores & Av. Alfredo Benavides 2315 & 4 de setiembre de 2019 & $9: 00$ a. m.-12:00 m. \\
\hline 9 & La Victoria & Av. Aviación, cdra. 18 & 12 de setiembre de 2019 & 9:00 a. m.-12:00 m. \\
\hline
\end{tabular}

Fuente: División de Calidad del Aire y Evaluaciones de la MML. Entrega directa.

\section{Instrumentos}

Se utilizó un analizador de gases portátil de marca Kane Automotive de clase 1, modelo Auto 4-1/MID. El equipo pertenece a la empresa Touring y Automóvil Club del Perú y cuenta con la calibración vigente. Los parámetros que evalúa son $\mathrm{CO}$ (\% vol.), $\mathrm{CO}_{2}$ (\% vol.), $\mathrm{HC}(\mathrm{ppm})$ y $\mathrm{O}_{2}$ (\% vol.).

\section{Métodos}

\section{a. Procedimiento en campo}

El procedimiento empezó con la instalación del stand de medición en los puntos (ver tabla 2). Se contó con el apoyo de inspectores municipales de transporte, quienes invitaban a los conductores de vehículos livianos (categoría M) a participar de la campaña. El personal de la MML les solicitó el CITV y tomó los siguientes datos: placa, marca, modelo, año de fabricación del vehículo, tipo de combustible, centro de ITV, vigencia del CITV y la información registrada en los parámetros CO (\% vol.), $\mathrm{CO}_{2}$ (\% vol.) + $\mathrm{CO}(\%$ vol.), $\mathrm{HC}$ (ppm), además del tipo de vehículo (taxi o particular).

Luego, el personal técnico de la empresa Touring y Automóvil Club del Perú colocó la sonda del equipo en el tubo de escape del vehículo detenido pero encendido, a la mínima cantidad de revoluciones (ralentí). El voucher impreso por el equipo con los resultados de la medición fue entregado a cada conductor.

\section{b. Análisis de datos}

Se procedió a organizar mediante Excel 2016 e IBM SPSS Statistics los datos ofrecidos por la División de Calidad del Aire y Evaluaciones de la MML. Para el diagnóstico se consideraron dos dimensiones: cumplimiento de la normativa vigente (en relación con los límites máximos permisibles vigentes) y gobernanza (en relación con la efectividad del control de las ITV).
En la primera dimensión se analizaron dos indicadores: el resultado total de la campaña en cuanto al cumplimiento de la normativa y el resultado de ese cumplimiento por tipo de vehículo (taxi o particular). Los datos se completaron con el portal web para consultar placas de la Gerencia de Transporte Urbano. Los vehículos considerados aprobados no superaron los límites máximos permisibles y los desaprobados incumplieron, al menos, un parámetro de los límites máximos permisibles. Ambos indicadores se expresaron en porcentaje considerando los 327 vehículos muestreados.

Posteriormente se identificó el número de veces que cada parámetro excedió los límites máximos permisibles. A continuación, se analizaron las medias obtenidas para cada parámetro del total de la campaña y las medias obtenidas por distrito. La comparación de medias se realizó usando Anova de un factor y la prueba post hoc de Tukey.

La segunda dimensión se enfocó en el sector de las empresas emisoras del CITV (empresas CITV) y analizó cuatro indicadores: cumplimiento de la ITV de los vehículos en circulación, efectividad de las ITV, empresas CITV más solicitadas y aprobación por empresa CITV. En el primer indicador se evaluó la cantidad de vehículos con CITV vigente, con CITV no vigente, con menos de cuatro años de antigüedad que no necesitaban CITV según el Decreto Supremo 006-2012-MTC y los que no contaban con CITV e incumplían la normativa. En el segundo indicador se evaluó la cantidad de aprobación real de los vehículos que contaban con el CITV vigente. En el tercer indicador se evaluó la cantidad de vehículos con el CITV (vigente o no vigente) por empresas CITV. En el cuarto indicador se evaluó, por empresa CITV, la cantidad de CITV vigentes en comparación con la cantidad de vehículos aprobados de esa muestra. 


\section{Resultados}

\section{Dimensión 1: Cumplimiento de la normativa vigente}

\section{Cumplimiento de la normativa vigente}

En la zona de Lima Sur se ejecutó la campaña en los distritos de Villa María del Triunfo, con una muestra de 9 vehículos, de los cuales 1 aprobó y 8 desaprobaron, y en Villa El Salvador, donde se midió a 32 vehículos, de los cuales 12 aprobaron y 20 desaprobaron.

En la zona de Lima Centro se ejecutó la campaña en los distritos de Jesús María, con una muestra de 26 vehículos, de los cuales 5 aprobaron y 21 desaprobaron; San Isidro, con una muestra de 20 vehículos, de los cuales 3 aprobaron y 17 desaprobaron; Lima Cercado, con una muestra de 38 vehículos, de los cuales 10 aprobaron y 28 desaprobaron;

a)

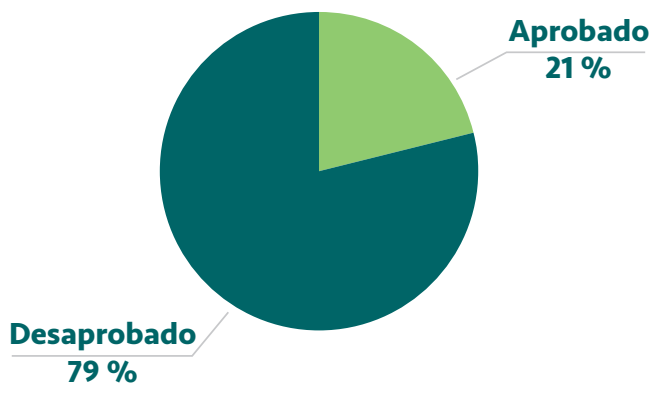

Miraflores, con una muestra de 31 vehículos, de los cuales 7 aprobaron y 24 desaprobaron; La Victoria, con una muestra de 41 vehículos, de los cuales 11 aprobaron y 30 desaprobaron; y Surquillo, con una muestra de 54 vehículos, de los cuales 6 aprobaron y 48 desaprobaron.

En la zona de Lima Este se ejecutó la campaña de medición de emisiones de $\mathrm{CO}$ (\% vol), $\mathrm{CO}_{2}(\%$ vol) y $\mathrm{HC}$ (ppm) en Santa Anita, con una muestra de 35 vehículos, de los cuales 8 aprobaron y 27 desaprobaron, y en La Molina, con una muestra de 41 vehículos, de los cuales 6 aprobaron y 35 desaprobaron.

La campaña contó con la participación de 327 vehículos, de los cuales 69 aprobaron y 259 desaprobaron la normativa vigente (ver figura 1a), lo que representa el $21 \%$ y el $79 \%$, respectivamente.

b)

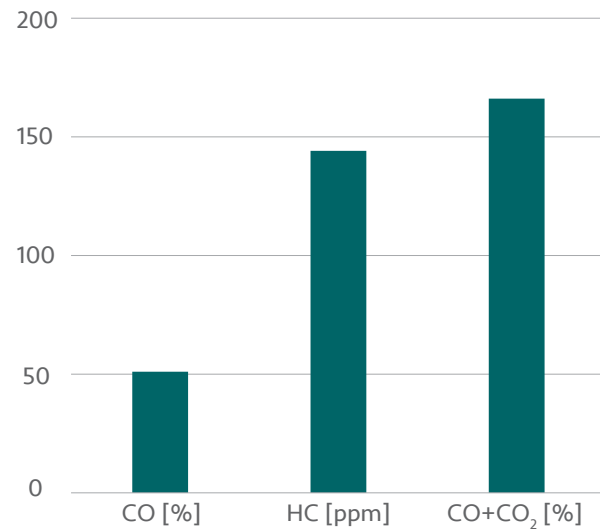

Figura 1. a) Porcentaje de desaprobados y aprobados de la campaña. Se muestra la proporción de vehículos que aprobaron y desaprobaron la normativa vigente. b) Gráfica de parámetros (gases). Se muestra el número de veces que los parámetros evaluados exceden la normativa vigente.

Tabla 3. Medias obtenidas por parámetro y distrito*

\begin{tabular}{cccc}
\hline Distrito & $\mathbf{C O}(\%)$ & $\mathbf{C O}+\mathbf{C O}_{\mathbf{2}}(\%)$ & HC (ppm) \\
\hline $\begin{array}{c}\text { Villa María del } \\
\text { Triunfo }\end{array}$ & $0,95 \pm 0,40$ & $7 \pm 1,64^{\mathrm{ab}}$ & $458 \pm 84,91^{\mathrm{ab}}$ \\
\hline Jesús María & $0,21 \pm 0,09$ & $8,39 \pm 0,93^{\mathrm{ab}}$ & $1325 \pm 598,87^{\mathrm{a}}$ \\
\hline San Isidro & $0,26 \pm 0,10$ & $6,99 \pm 0,99^{\mathrm{ab}}$ & $271 \pm 95,51^{\mathrm{ab}}$ \\
\hline Santa Anita & $0,24 \pm 0,13$ & $6,41 \pm 0,65^{\mathrm{b}}$ & $78 \pm 30,06^{\mathrm{b}}$ \\
\hline La Molina & $0,66 \pm 0,29$ & $7,33 \pm 0,75^{\mathrm{ab}}$ & $260 \pm 110,55^{\mathrm{b}}$ \\
\hline Villa El Salvador & $0,33 \pm 0,13$ & $8,69 \pm 0,91^{\mathrm{ab}}$ & $685 \pm 312,16^{\mathrm{ab}}$ \\
\hline Surquillo & $0,67 \pm 0,21$ & $6,43 \pm 0,60^{\mathrm{b}}$ & $809 \pm 233,17^{\mathrm{ab}}$ \\
\hline Miraflores & $0,09 \pm 0,03$ & $6,56 \pm 0,74^{\mathrm{ab}}$ & $210 \pm 74,62^{\mathrm{b}}$ \\
\hline La Victoria & $0,86 \pm 0,28$ & $9,91 \pm 0,63^{\mathrm{a}}$ & $272 \pm 60,95^{\mathrm{b}}$ \\
\hline Cercado de Lima & $0,44 \pm 0,18$ & $7,17 \pm 0,99^{\mathrm{ab}}$ & $60 \pm 25,56^{\mathrm{b}}$ \\
\hline
\end{tabular}

* Se usó la prueba de Tukey para determinar las diferencias significativas de medias $(p<0,05)$. Se indica el error estándar en cada media. Los grupos denominados $a$ y $b$, que representan a los distritos, tienen diferencias significativas entre sí. Entre el resto de los grupos no existen diferencias significativas.
Tabla 4. Análisis de la varianza con un factor Anova por distrito

\begin{tabular}{ccc}
\hline Parámetro & Nivel de confianza & Significancia \\
\hline $\mathrm{HC}$ & $5 \%$ & 0,002 \\
\hline $\mathrm{CO}$ & $5 \%$ & 0,129 \\
\hline $\mathrm{CO}+\mathrm{CO}_{2}$ & $5 \%$ & 0,018
\end{tabular}

Nota: hay diferencias significativas en la emisión de gases según los distritos.

Tabla 5. Análisis de la varianza con un factor Anova por tipo de vehículo

\begin{tabular}{ccc}
\hline Parámetro & Nivel de confianza & Significancia \\
\hline $\mathrm{HC}$ & $5 \%$ & 0,21 \\
\hline $\mathrm{CO}$ & $5 \%$ & 0,13 \\
\hline $\mathrm{CO}+\mathrm{CO}_{2}$ & $5 \%$ & 0,7 \\
\hline
\end{tabular}

Nota: no hay diferencias significativas en las emisiones de gases según el tipo de uso de vehículo. 


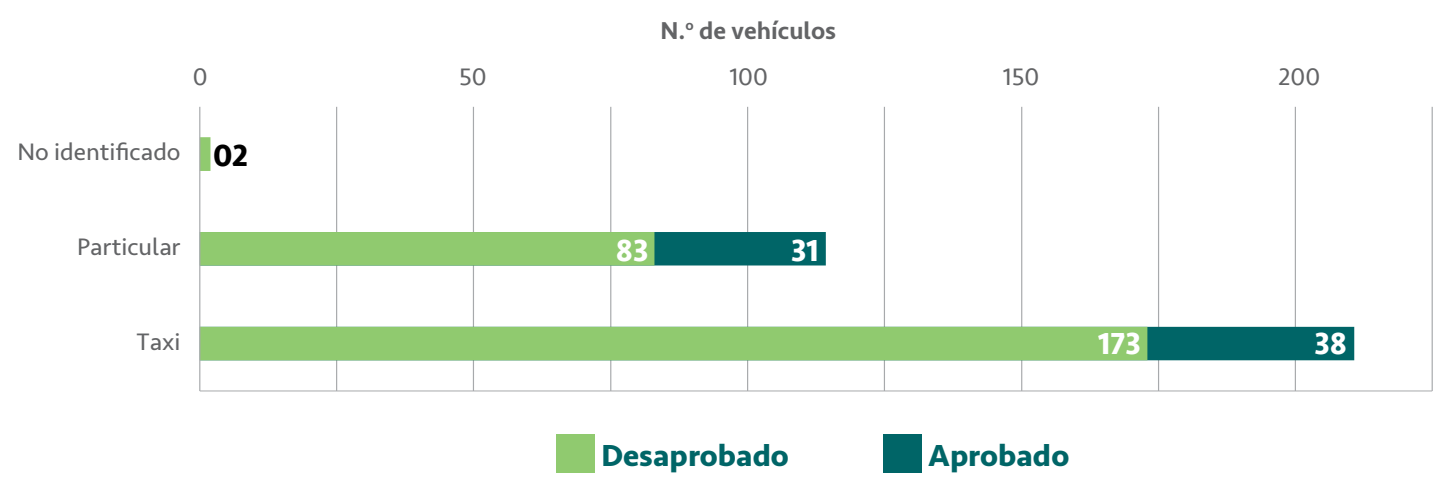

Figura 2. Relación entre el tipo de vehículo y el cumplimiento de la normativa. Se muestra la cantidad de vehículos aprobados y desaprobados según su tipo de uso.

\section{Cumplimiento de la normativa vigente en relación con el tipo de vehículo}

En la campaña ejecutada se contó con la participación de vehículos de uso privado y del servicio de taxi. De los 327 vehículos evaluados 211 pertenecían a la categoría de taxi, de los cuales solo aprobó el 17,5 \% (38 vehículos) y desaprobó el 82,5 \% (173 vehículos). A su vez, 114 pertenecían a la categoría de uso particular, de los cuales solo aprobó el 27,2 \% (31 vehículos) y desaprobó el 72,2 \% (83 vehículos). Los dos vehículos cuya categoría no se logró identificar salieron desaprobados (ver figura 2).

\section{Dimensión 2: Gobernanza}

\section{Cumplimiento de la ITV de los vehículos en circulación}

En la campaña ejecutada se solicitó los CITV a los vehículos participantes. De los 327 vehículos evaluados, 237 contaban con el CITV vigente, 17 contaban con el CITV vencido, 58 vehículos tenían entre 0 a 4 años de fabricación (por lo cual se les exoneró de la necesidad de tener un CITV) y 15 vehículos no contaban con el CITV (ver figura 3).

\section{Efectividad de las ITV}

De los 327 vehículos evaluados, 237 contaban con el CITV vigente, y de ellos solo el 16 \% (37 vehículos) aprobó los parámetros en la medición de la campaña. El 84 \% (200 vehículos) no aprobó los parámetros evaluados, al no coincidir con el estado que indicaba el CITV (ver figura 4).

\section{Empresas CITV más solicitadas}

Del total de la campaña, se observó que 70 vehículos obtuvieron el CITV por la empresa Farenet, 48 por RTP, 38 por Jardel Perú, 19 por Lidercon, 15 por Revisa Perú, 20 por Hersa y 44 por otras empresas (ver figura 5).

Teniendo en cuenta que Farenet, RTP y Jardel Perú encabezan la preferencia de los conductores para la obtención del CITV, se procedió a evaluar la cantidad de vehículos que desaprobaron la evaluación, con el fin de validar el CITV emitido.

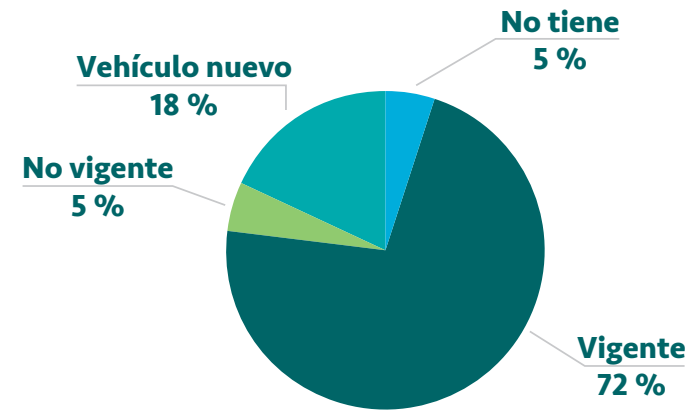

Figura 3. Cumplimiento de ITV de los vehículos muestreados. Se muestra la cantidad de vehículos que cuentan con CITV y los que aún están vigentes, es decir, tienen permiso de circular.

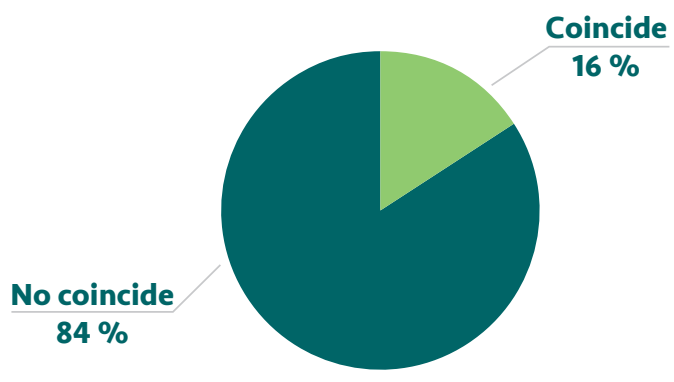

Figura 4. Relación de vehículos aprobados con vehículos con ITV vigente. Se muestra la cantidad de vehículos que aprobaron y no aprobaron (coincide y no coincide, respectivamente) los parámetros evaluados, pese a contar con CITV vigente.

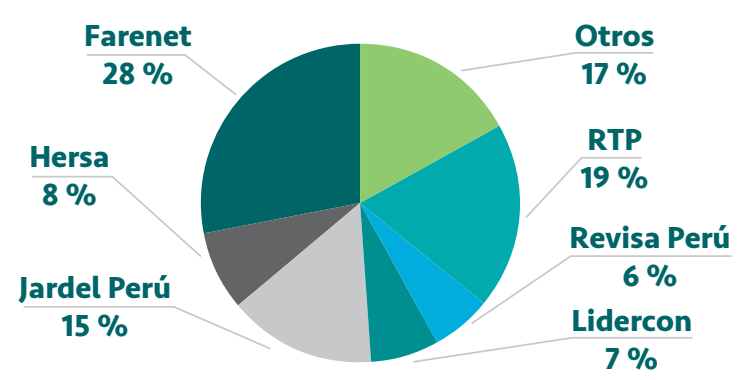

Figura 5. Empresa preferida por los vehículos en certificar la ITV. Se muestra la distribución de CITV emitidos de los vehículos que cuentan con CITV vigente y no vigente. 
Aprobación por empresa CITV

\section{a. Farenet}

De los 66 vehículos certificados en estado vigente por la empresa Farenet, solo 12 aprobaron la evaluación de la campaña.

\section{b. Revisiones Técnicas del Perú}

De los 46 vehículos certificados en estado vigente por la empresa Revisiones Técnicas del Perú (RTP), solo 7 aprobaron la evaluación.

\section{c. Jardel Perú}

De los 35 vehículos certificados en estado vigente por la empresa Jardel Perú (RTP), solo 3 aprobaron la evaluación.

\section{Discusión}

El $79 \%$ de los vehículos evaluados en la campaña incumplen la normativa establecida en el Decreto Supremo 010-2017-MINAM. La gran mayoría cuenta con el CITV vigente, lo que muestra la falta de veracidad de los datos. Esta situación puede deberse tanto a las malas condiciones técnicas de los vehículos como a la desfasada metodología de evaluación de nuestra normativa. El método de la normativa vigente consta de una verificación de los niveles de emisión de cuatro gases en prueba estática. Según Galván, Melo y Alcântara de Vaconcellos (2014), esta metodología es caduca porque no representa adecuadamente a los vehículos modernos, que están equipados con catalizadores e inyectores electrónicos. Es decir, tenemos un programa de ITV, basado en la medición de las emisiones en prueba estática, que aprueba y reprueba vehículos al azar.

El parámetro que más incumple la normativa en esta investigación es el $\mathrm{CO}+\mathrm{CO}_{2} \%$, que representa 166 incumplimientos, seguido de los 144 incumplimientos del parámetro $\mathrm{HC}$ (ppm). Esta situación expone la combustión incompleta de los motores vehiculares, que da lugar a las altas emisiones de monóxido de carbono e hidrocarburos no quemados. Nuestra normativa considera la suma de monóxido de carbono con dióxido de carbono como un parámetro mínimo. Esta metodología está destinada a antiguos motores carburadores, dado que mientras hay más porcentaje de emisión de dióxido de carbono, existe una combustión más completa en el motor y disminuye la emisión de hidrocarburos no quemados.

Se comprobó que existe una diferencia significativa en las emisiones vehiculares según el distrito. Destaca Jesús María en relación con el parámetro $\mathrm{HC}$ (ppm), al igual que Villa María del Triunfo, San Isidro, Villa El Salvador y Surquillo.

Las tres empresas de ITV más usadas por los vehículos también son las que tienen más porcentaje de desaprobados en la normativa, a pesar de tener el certificado vigente.

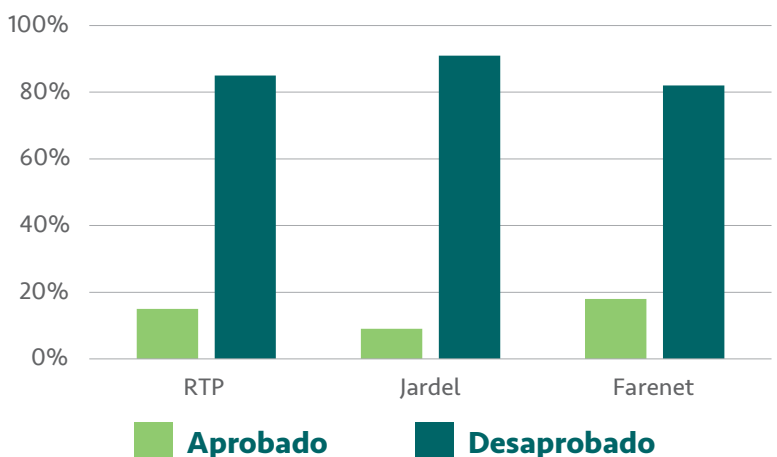

Figura 6. Coincidencia de aprobación de vehículos por empresa. Se muestra el porcentaje de vehículos aprobados y desaprobados de los que cuentan con el CITV vigente de las tres empresas que lideran la cantidad de CITV emitidos.

Se recomienda la revisión de la metodología de evaluación de emisiones vehiculares, para desarrollar nuevos procesos de diagnóstico más asertivos en representar las emisiones de vehículos modernos que cuentan con inyectores electrónicos y catalizadores. En Ciudad de México se utiliza una metodología especial para vehículos más modernos, que mide las emisiones de un vehículo con el método dinámico con carga y simula las condiciones reales de uso, ya que el vehículo rueda sobre rollos. Este método también es utilizado en Santiago de Chile, con la ayuda de un dinamómetro (Galván, Melo y Alcântara de Vaconcellos, 2014). Actualmente se desarrollan metodologías más avanzadas y cada vez más fiables de diagnóstico de emisiones vehiculares. Entre ellas están las redes neuronales artificiales (RNA), que predicen las emisiones de monóxido de carbono e hidrocarburos sin quemar, ocasionados por fallas mecánicas en el motor (Contreras, León y Maldonado, 2020).

\section{Conclusión}

La desfasada metodología de análisis de medición de gases de nuestra normativa (Galván, Melo y Alcântara de Vaconcellos, 2014) y la ineficiencia en el control del Sistema Nacional de ITV se ven representadas en el alto porcentaje de vehículos desaprobados. Además, se identifica como uno de los mayores problemas la combustión incompleta en los motores por las altas emisiones de hidrocarburos no quemados.

\section{Agradecimientos}

El presente artículo se publica gracias a la información otorgada por Anderson Huayna Castro, jefe de la división de Calidad del Aire y Evaluaciones Ambientales de la Municipalidad Metropolitana de Lima, y la empresa Touring y Automóvil del Perú, que solicitaron el análisis de sus datos obtenidos en la campaña «Respira Limpio». 


\section{Fuente de financiamiento}

Este trabajo fue autofinanciado.

\section{Contribución de autoría}

CP-J contribuyó en la gestión de la información, el análisis de los resultados y la redacción del artículo. JV-C contribuyó en el procesamiento de la información, el análisis de los resultados y la redacción. VB-S contribuyó en el asesoramiento en la metodología de investigación. Todos los autores aprobaron la versión final del texto.

\section{Potenciales conflictos de interés}

Los autores declaran que no existe ningún conflicto de interés.

\section{Referencias bibliográficas}

Caminos, J., Enrique, C., Ghirardi, R., Graizaro, A., Rusillo, S. y Pacheco, C. (S. f.). Calidad de aire en la ciudad de Santa Fe. Disponible en: http://www.edutecne.utn.edu.ar/monografias/calidad aire Santa_Fe.pdf

Contreras Urgilés, W., León Japa, R. y Maldonado Ortega, J. (2020). «Predicción de emisiones de $\mathrm{CO}$ y $\mathrm{HC}$ en motores Otto mediante redes neuronales». Ingenius. Revista de Ciencia y Tecnología, 23, 3039. DOI: https://doi.org/10.17163/ings.n23.2020.03

Galván, A., Melo, O. y Alcântara de Vaconcellos, E. (2014). Inspección técnica vehicular en América Latina. Buenos Aires: CAF. Disponible en: http://scioteca.caf.com/handle/123456789/793

Gerencia de Transporte Urbano, Municipalidad Metropolitana de Lima. (2020). «Consulta de placas». Disponible en: http:// www.sistemasgtu.munlima.gob.pe/Internet_Web/ConsultaGTU/ ConsultaGTU.aspx

Macke, J., Rubim Sarate, J. A. y De Atayde Moschen, S. (2019). «Smart sustainable cities evaluation and sense of community». Journal of Cleaner Production, 239, 118103. Disponible en: https://doi. org/10.1016/j.jclepro.2019.118103

Ministerio de Transportes y Comunicaciones. (2020). «Consulta de CITV». Disponible en: https://portal.mtc.gob.pe/reportedgtt/form/ frmconsultaplacaitv.aspx

ONU. (2018). «Ciudades y comunidades sostenibles». Disponible en: https://www.un.org/sustainabledevelopment/es/cities/

Posada, C. (2018). «Aumento continuo del parque automotor, un problema que urge solucionar». La Cámara, 816, pp. 24-26. Disponible en: https://www.camaralima.org.pe/RepositorioAPS/0/0/par/ EDICION816/edicion816.pdf

Presidencia de la República. (29 de junio de 2012). Decreto Supremo 006-2012-MTC. Diario Oficial El Peruano. Disponible en: http:// www.perutransportes.com/ficheros/DS-006-2012-MTC.pdf

Presidencia de la República. (31 de octubre de 2001). Decreto Supremo 047-2001-MTC. Diario Oficial El Peruano. Disponible en: https:// portal.mtc.gob.pe/transportes/socioambientales/documentos\%20 normas\%20legales/DS_047.pdf

Presidencia de la República. (30 de noviembre de 2017). Decreto Supremo 010-2017-MINAM. Diario Oficial El Peruano. Disponible en: https://sinia.minam.gob.pe/normas/establecen-limites-maximospermisibles-lmp-emisiones-atmosfericas
Presidencia de la República. (8 de setiembre de 2017). Decreto Supremo 025-2017-EM. Establecen medidas relacionadas al contenido de azufre en el diésel, gasolina y gasohol para su comercialización y uso. Ministerio de Energías y Minas. Diario Oficial El Peruano. Disponible en: https://busquedas.elperuano.pe/ normaslegales/establecen-medidas-relacionadas-al-contenido-deazufre-en-el-decreto-supremo-n-025-2017-em-1563113-4/

Vintimilla Jarrín, P. (2015). Análisis de resultados de la medición de emisiones de gases contaminantes de fuentes móviles a partir de la implementación de la revisión técnica vehicular en el cantón de Cuenca (tesis para obtener el grado de ingeniero mecánico automotriz). Universidad Politécnica Salesiana, Cuenca. Disponible en: https:// dspace.ups.edu.ec/bitstream/123456789/7647/1/UPS-CT004530. pdf

Zucchetti, A. y Freundt, D. (2018). Ciudades del Perú. Primer reporte nacional de indicadores urbanos. 2018. Con un enfoque de sostenibilidad y resiliencia. Lima: Periferia y WWF. Disponible en: https://d2ouvy59p0dg6k.cloudfront.net/downloads/ciudades sostenibles_1.pdf 